\title{
ERRATUM
}

doi:10.1038/nature08660

\section{Direct inhibition of the NOTCH transcription factor complex}

Raymond E. Moellering, Melanie Cornejo, Tina N. Davis, Cristina Del Bianco, Jon C. Aster, Stephen C. Blacklow, Andrew L. Kung, D. Gary Gilliland, Gregory L. Verdine \& James E. Bradner

\section{Nature 462, 182-188 (2009)}

In the print issue of this Article, text from the last line of the Figure 4 legend, defining the scale bar lengths, is inadvertently missing. This sentence should read "Scale bars, $50 \mu \mathrm{m}$ ". 